\title{
Association analysis of $D T D 1$ gene variations with aspirin-intolerance in asthmatics
}

\author{
CHARISSE FLERIDA A. PASAJE ${ }^{1 *}$, JOON SEOL BAE $^{1 *}$, BYUNG-LAE PARK $^{2}$, AN-SOO JANG $^{3}$, \\ SOO-TAEK UH ${ }^{4}$, MI-KYEONG KIM ${ }^{5}$, IN SONG KOH ${ }^{6}$, JEONG-HYUN KIM ${ }^{1}$, TAE-JOON PARK ${ }^{1}$, \\ JIN-SOL LEE ${ }^{1}$, YONGHA KIM ${ }^{1}$, CHOON-SIK PARK ${ }^{3}$ and HYOUNG DOO SHIN ${ }^{1,2}$ \\ ${ }^{1}$ Department of Life Science, Sogang University, Seoul 121-742; ${ }^{2}$ Department of Genetic Epidemiology, \\ SNP Genetics, Inc., Seoul 153-803; ${ }^{3}$ Division of Allergy and Respiratory Medicine, Soonchunhyang \\ University Seoul Hospital, Seoul 140-743; ${ }^{4}$ Genome Research Center for Allergy and Respiratory \\ Diseases, Division of Allergy and Respiratory Medicine, Soonchunhyang University Bucheon \\ Hospital, Bucheon 420-767; ${ }^{5}$ Department of Internal Medicine, Chungbuk National University, \\ College of Medicine, Cheongju, Chungcheongbuk-do 361-711; ${ }^{6}$ Department of Physiology, \\ College of Medicine, Hanyang University, Seoul 133-791, Republic of Korea
}

Received January 20, 2011; Accepted March 14, 2011

DOI: $10.3892 / \mathrm{ijmm} .2011 .669$

\begin{abstract}
Aspirin ingestion is a common precipitating factor of life-threatening asthma attacks, requiring some patients to undergo mechanical ventilation. The gene, D-tyrosyl-tRNA deacylase 1 (DTD1), may be a risk factor for aspirin-intolerant asthma (AIA) by catalyzing the hydrolysis of D-tryptophan and interacting with the tyrosyl-tRNA synthetase (tyrRS) enzyme, which promotes a pro-inflammatory phenotype. In order to investigate the association of DTDl variants with the risk of AIA in an asthma cohort, 38 single nucleotide polymorphisms (SNPs) were genotyped and 5 major haplotypes were obtained in 163 AIA cases and 429 aspirin-tolerant asthma (ATA) controls. Differences in DTD1 SNP and haplotype distributions were analyzed using logistic and multiple regression models and were adjusted for age, gender, smoking status, atopy and body mass index (BMI) as covariates. Subsequent analyses revealed no association between DTDI variants and the risk of AIA. Although nominal evidence of an association was detected between several DTDl variants and the rate of decline of the forced expiratory volume in the first second $\left(\mathrm{FEV}_{1}\right)$ in AIA patients (rs6136444, rs6136469,
\end{abstract}

Correspondence to: Dr Hyoung Doo Shin, Department of Life Science, Sogang University, Seoul 121-742, Republic of Korea E-mail: hdshin@sogang.ac.kr

Dr Choon-Sik Park, Division of Allergy and Respiratory Medicine, Soonchunhyang University Seoul Hospital, Seoul 140-743, Republic of Korea

E-mail: schalr@schbc.ac.kr

${ }^{*}$ Contributed equally

Key words: aspirin-intolerant asthma, D-tyrosyl-tRNA deacylase 1, single nucleotide polymorphism, haplotype rs6081338 and DTD1_ht5; $\mathrm{P}=0.01-0.02)$, the signals reached the threshold of multiple testing corrections, suggesting that DTDl variants do not affect the abnormalities of the upper airways in AIA patients.

\section{Introduction}

Aspirin-intolerant asthma (AIA) is the development of bronchoconstriction in asthmatic patients following the ingestion of aspirin, a non-steroidal anti-inflammatory drug (NSAID) $(1,2)$, and is characterized by the triad of chronic rhinosinusitis, nasal polyps and exacerbated asthma akin to prolonged viral respiratory infection (2). The prevalence rate of aspirin intolerance has been reported to be around 10-20\% among adult asthmatics $(3,4)$, with an observed predominance among women $(5,6)$. A previous study has reported that $25 \%$ of asthma patients who required emergency mechanical ventilation are aspirin intolerant (7), hence, ingestion of aspirin may be a precipitating factor in life-threatening asthma attacks (8). However, despite the well-defined clinical characteristics of the disease, genetic underpinnings of AIA pathogenesis are still unclear.

The etiology of AIA development is attributable to the combinatorial effects of environmental and genetic risk factors. We have previously identified several variants in the solute carrier family 6 (neurotransmitter transporter, betaine/ GABA) member 12 (SLC6A12), the emilin/multimerin domain-containing protein 2 (EMID2) and the fibrous sheath interacting protein 1 ( $F S I P 1)$ genes that are risk factors of AIA susceptibility (9-11), suggesting that complex genetic mechanisms underlie AIA pathogenesis. Although candidate gene-association studies and genome-wide association studies (GWAS) have provided unprecedented insights into the triggers and pathophysiology of asthma, the exact functional mechanisms of AIA development are still unclear and the roles of numerous candidate genes still need to be elucidated. 
The human D-tyrosyl-tRNA deacylase 1 (DTDI) gene on chromosome 20p11.23 is a cellular component of the cytoplasm and is expressed mainly in the testis, ovary, spleen and in the adult and fetal brain (12). The protein encoded by this gene interacts with tyrosyl-tRNA synthetase (tyrRS) by catalyzing the hydrolysis of D-tyrosyl-tRNA, thereby preventing the misacylated accumulation of metabolically inactive tRNA molecules including several D-amino acids such as D-tyrosine, D-aspartic acid and D-tryptophan $(13,14)$. During the activation of cellular immune responses, interferon (IFN)- $\gamma$ induces the enzyme indoleamine 2,3-dioxygenase (IDO), which catalyzes tryptophan into kynurenine and serotonin (15), resulting in inhibition of T-cell proliferation (16) and increased severity of allergic asthma (17). Elevated levels of serum kynurenine and serotonin in individuals with various degrees of chronic airway obstruction compared to non-asthmatic controls provide evidence that tryptophan may play a role in asthma pathogenesis (18-20). In particular, inactivation or misregulation of the DTDl gene, may hamper the recycling of misacylated tRNA molecules and lead to an increase in the toxicity of D-amino acids including tryptophan (21).

With the crucial role of DTD1 in inhibiting the harmful effects of D-tryptophan accumulation as well as its association with the pro-inflammatory promoting tyrRS, we hypothesized that genetic variations in DTD1 may influence bronchial hypersensitivity in AIA patients. To elucidate the association between DTDI variants and the risk of AIA, a case-control analysis was carried out in a Korean population.

\section{Materials and methods}

Study subjects. Asthma patients were recruited from nine Korean hospitals belonging to the Asthma Genome Research Center, to serve as the primary subjects for our study. Each patient, as diagnosed by trained physicians, showed clinical symptoms that met the criteria for asthma according to the Global Initiative for Asthma (GINA) (22). Evaluation of the subjects included dyspnea and wheezing during the past year plus one of the following: i) airway reversibility measured by a positive bronchodilator response of a $>15 \%$ increase in the forced expiratory volume in the first second $\left(\mathrm{FEV}_{1}\right)$ or a $>12 \%$ increase in $\mathrm{FEV}_{1}$ plus $200 \mathrm{ml}$ following inhalation of a short-acting bronchodilator; ii) airway hyperreactivity to $<10 \mathrm{mg} / \mathrm{ml} \mathrm{PC}_{20}$ methacholine; or iii) $>20 \%$ increase in $\mathrm{FEV}_{1}$ following 2 weeks of treatment with inhaled steroids and long-acting bronchodilators (23). Twenty-four common inhalant allergens (e.g., dust mites, cat fur, dog fur, cockroaches, grasses, trees, ragweed pollen; Bencard Co. Ltd., Brentford, UK) were used in a skin-prick test. Total immunoglobulin E (IgE) was measured using the CAP system (Pharmacia Diagnostics, Uppsala, Sweden). Atopy was defined as a wheal reaction $\geq$ to histamine or than $3 \mathrm{~mm}$ in diameter. Pulmonary function tests were performed using the Vmax Series 2130 Autobox Spirometer (SensorMedics, Yorba Linda, CA) with adherence to the American Thoracic Society (ATS) guidelines (24). The reference values of lung functions used were according to the Morris-Polgar standards $(25,26)$. All asthmatics underwent oral aspirin challenge (OAC) that was performed with increasing doses of aspirin $(10-450 \mathrm{mg})$ $(27,28)$ with modifications. The subjects reported no increase in asthma symptoms or respiratory tract infections within 6 weeks prior to the test. Briefly, patients with a history of aspirin hypersensitivity were given $30 \mathrm{mg}$ and those having no history of aspirin hypersensitivity were initiated with $100 \mathrm{mg}$ of aspirin orally. Symptoms, external signs (urticaria and angioedema) and $\mathrm{FEV}_{1}$ were documented every $30 \mathrm{~min}$ for a period of $2 \mathrm{~h}$. In the absence of any symptoms or signs suggestive of an adverse reaction after $2 \mathrm{~h}, 60$ or $100 \mathrm{mg}$ of aspirin was administered and the same measurements were repeated every $1 \mathrm{~h}$, increasing the doses up to $450 \mathrm{mg}$ until the patient developed a reaction. If no reaction occurred $5 \mathrm{~h}$ after the final dose, the test was deemed negative. Changes in the $\mathrm{FEV}_{1}$ were followed for $5 \mathrm{~h}$ after the final aspirin dose. Aspirin-induced bronchospasm, reflected by the rate (\%) of decline in $\mathrm{FEV}_{1}$, was calculated as the pre-challenge $\mathrm{FEV}_{1}$ minus the post-challenge $\mathrm{FEV}_{1}$ divided by the pre-challenge $\mathrm{FEV}_{1}$. Categorization of patients was based on individual OAC reactions. Asthmatics exhibiting $\geq 20 \%$ decrease in $\mathrm{FEV}_{1}$ or a $15-19 \%$ decrease in $\mathrm{FEV}_{1}$ with naso-ocular or cutaneous reactions were diagnosed as AIA cases, whereas those demonstrating $<15 \%$ decrease in $\mathrm{FEV}_{1}$ without naso-ocular or cutaneous reactions were identified as aspirin-tolerant asthma (ATA) controls. This study was undertaken with the understanding and written consent of each subject, and the protocols were approved by the Institutional Review Board of each hospital.

SNP genotyping and haplotype construction. Candidate polymorphic SNPs of the DTD1 gene were selected and screened from the International HapMap Project (http://hapmap.ncbi. nlm.nih.gov/) based on the minor allele frequencies (MAF) in the Asian population (Han Chinese and Japanese), the linkage disequilibrium (LD) status, and the importance of the position in the gene. Genomic DNA was extracted from peripheral blood lymphocytes using the Gentra Puregene kit (Gentra Systems, Minneapolis, MN) according to the manufacturer's protocol. SNP genotyping was performed using TaqMan assay (29) in the ABI PRISM 7900HT sequence detection system (Applied Biosystems, CA, USA). The genotyped data quality was assessed by duplicate DNA checking $(n=10$; rate of concordance in duplicates $>99 \%$ ). Using the Phase algorithm v.2.0 software (30), haplotypes were inferred from the successfully genotyped SNPs and those with a frequency $>0.05$ were included in the association analyses.

Statistical analyses. The LD between all pairs of biallelic loci were determined by Lewontin's $\mathrm{D}^{\prime}\left(\left|D^{\prime}\right|\right)$ and the LD coefficient $r^{2}$-values were examined using the Haploview algorithm (31). To determine the association between the DTD1 genotype distributions in AIA cases and ATA controls, the odds ratios and 95\% confidence intervals as well as the corresponding P-values were calculated using logistic regression analysis controlling for age (continuous variable), gender $($ male $=0$, female $=1$ ), smoking status (non-smoker $=0$, ex-smoker $=1$, smoker $=2$ ), atopy (absence $=0$, presence $=1$ ) and body mass index (BMI) as covariates to eliminate or reduce any confounding variables that might influence the findings. Data was managed and analyzed using the Statistical Analysis System (SAS) version 9.1 (SAS Inc., Cary, NC). In addition, the differences in the decline rate of $\mathrm{FEV}_{1}$ following aspirin 
Table I. Clinical profiles of the study subjects $(n=592)$.

\begin{tabular}{lccc}
\hline Clinical profile & Asthmatics (all subjects) & AIA & ATA \\
\hline Number of subjects, $\mathrm{n}$ & 592 & 163 & 429 \\
Age, years, mean (range) & $46.15(15.40-77.88)$ & $43.13(17.22-72.73)^{\mathrm{a}}$ & $47.30(15.40-77.88)$ \\
Gender, $\mathrm{n}$ (male/female) & $206 / 386$ & $59 / 104$ & $147 / 282$ \\
$\%$ Smokers, (current/ex-smokers) & $27.70(12.50 / 15.20)$ & $21.47(12.88 / 8.59)^{\mathrm{a}}$ & $30.07(12.35 / 17.72)$ \\
Height, cm & $160.78 \pm 8.63$ & $161.72 \pm 8.69$ & $160.42 \pm 8.39$ \\
Weight, kg & $62.81 \pm 10.84$ & $61.25 \pm 10.38^{\mathrm{a}}$ & $63.40 \pm 10.97$ \\
Body mass index, kg/m ${ }^{2}$ & $24.24 \pm 3.39$ & $23.39 \pm 3.25^{\mathrm{a}}$ & $24.58 \pm 3.39$ \\
$\%$ FEV decline by aspirin provocation & $9.27 \pm 13.24$ & $24.63 \pm 16.11^{\mathrm{b}}$ & $3.54 \pm 4.85$ \\
$\%$ Blood eosinophils & $6.01 \pm 5.73$ & $5.96 \pm 5.21$ & $6.03 \pm 5.92$ \\
FEV,$\%$ predicted & $90.54 \pm 16.97$ & $90.35 \pm 14.04^{\mathrm{a}}$ & $91.66 \pm 16.87$ \\
PC ${ }_{20}$ methacholine, mg/ml & $6.43 \pm 8.67$ & $5.02 \pm 7.83^{\mathrm{a}}$ & $6.91 \pm 8.90$ \\
Total IgE, IU/ml & $357.65 \pm 604.09$ & $348.60 \pm 596.44$ & $361.00 \pm 607.56$ \\
Positive skin test, $\%$ & 56.42 & 52.76 & 57.81 \\
Positive nasal polyp, $\%$ & 33.83 & $57.89^{\mathrm{b}}$ & $51.92^{\mathrm{b}}$
\end{tabular}

Values are the means \pm SE. BMI, body mass index; AIA, aspirin-intolerant asthma; ATA, aspirin-tolerant asthma. ${ }^{\text {a }} \mathrm{P}<0.05$, ${ }^{\text {b }} \mathrm{P}<0.0001$, statistically significant differences between AIA and ATA patients.

challenge among DTD1 genotypes and haplotypes were examined using regression analysis.

To achieve optimal correction for multiple testing of markers representing SNPs in LD, the effective number of independent marker loci (29.1223) was calculated using the SNPSpD software (http:/genepi.qimr.edu.au/general/daleN/SNPSpD/), a program that is based on the spectral decomposition $(\mathrm{SpD})$ of matrices of pair-wise LD between markers (32).

\section{Results}

Among a total of 592 asthmatics recruited in our study, 163 subjects were identified as AIA cases and 429 subjects were categorized as ATA controls based on the results of the aspirin provocation test. Table I depicts the clinical characteristics of the study subjects. From the data obtained, it was observed that AIA patients had a lower BMI $\left(23.39 \pm 3.25 \mathrm{~kg} / \mathrm{m}^{2}\right)$ compared to the ATA controls $\left(24.58 \pm 3.39 \mathrm{~kg} / \mathrm{m}^{2}\right)$. Our findings also demonstrate a significant difference between the mean age of AIA patients (43.1 years) and that of the controls (47.3 years). In addition, results from the aspirin provocation test showed a significant increase in the aspirin-induced decline rate of $\mathrm{FEV}_{1}$ in AIA patients compared to that of ATA controls (24.63\% in AIA and 3.54\% in ATA; $\mathrm{P}<0.0001)$. The predicted $\% \mathrm{FEV}_{1}$, smoking status and $\mathrm{PC}_{20}$ methacholine values were significantly lower in AIA patients than in the ATA controls $(\mathrm{P}<0.05)$. Furthermore, the rates of nasal polyps in AIA patients $(57.89 \%)$ were significantly higher compared to ATA controls (26.06), suggesting that aspirin-intolerance can elicit meaningful deficits in the upper airways. A positive history of aspirin hypersensitivity was also found to be significantly prevalent in AIA patients $(51.92 \%)$ compared to ATA subjects $(6.00 \%)$.
With an average call rate of 99.9\%, 38 DTD1 SNPs were successfully genotyped in the asthma cohort of 592 Korean patients; 1 SNP was localized in intron 2 and 1 in exon 6, 3 in intron 3, 25 in intron 4 and 8 SNPs were positioned in intron 5 (Fig 1A and Table II). Results from the Hardy-Weinberg equilibrium (HWE) test showed no significant differences between the distribution of the observed genotypes and the expected distributions ( $P>0.05$; Table II). The MAF of each SNP is shown in Table II. Using the genotyped SNPs, five major haplotypes with frequencies $>0.05$ (Fig. 1B) were obtained and included in the association analyses. The haplotypes were contained in one LD block (Fig. 1C) that was established from pair-wise comparisons of the 38 genotyped SNPs.

Results from logistic analyses showed no significant association between $D T D 1$ variants and the risk of AIA in a Korean population (Table III). Since the decline rate of $\mathrm{FEV}_{1}$ induced by aspirin provocation is an important diagnostic marker of AIA, further association analysis with the DTDI genetic variants was performed using a regression model. Initial results revealed significant associations between three DTD1 SNPs (rs6136444, rs6136469 and rs6081338) and the decline rate of $\mathrm{FEV}_{1}$ via a recessive model of genetic inheritance $(\mathrm{P}=0.01-0.02$; Table IV), whereas one haplotype (DTD1_ht5) was also significantly associated with the decline rate of $\mathrm{FEV}_{1}$ via co-dominant and dominant mechanisms $(\mathrm{P}=0.02$; Table IV). However, with 29.1223 as the effective number of independent marker loci, the significant values were not retained after multiple testing corrections.

\section{Discussion}

The development of AIA can be attributed to combinatorial effects of genetic and environmental factors. In some patients, 
A

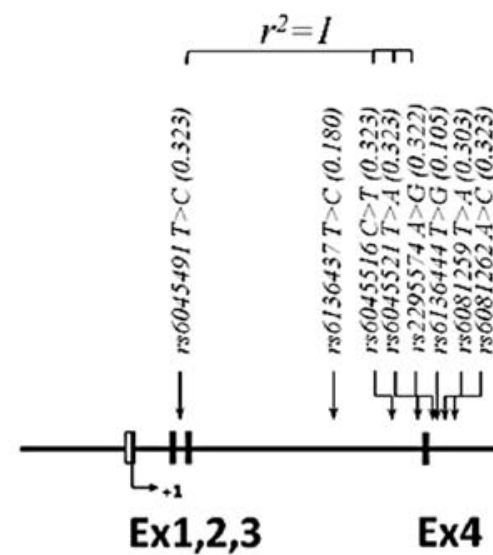

$\stackrel{r^{2}=1}{r^{2}=1} \stackrel{r^{2}=1}{r^{2}=1}$

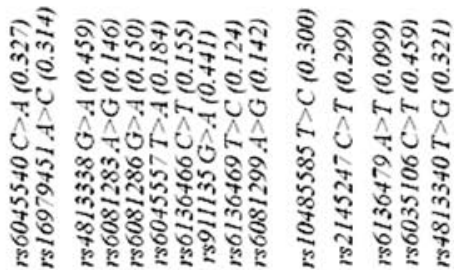

W

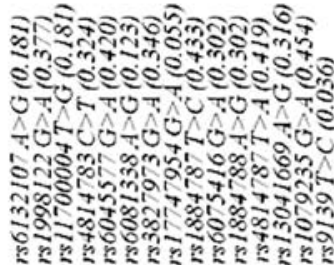

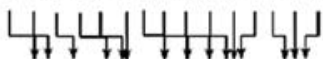

B

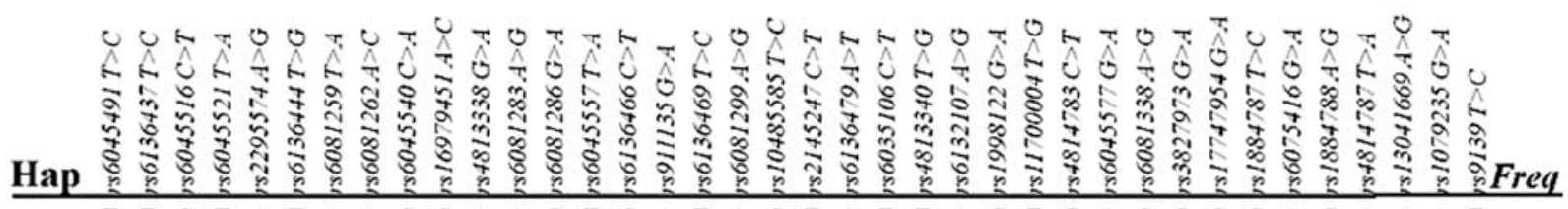

DTDI ht T T T C T A T T A A C C C A A G T C A T A C T A T T A G T C A G G G C A G A A A A T

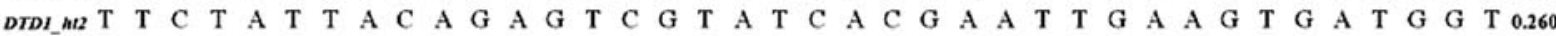

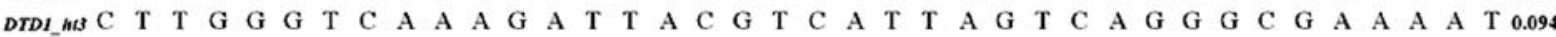

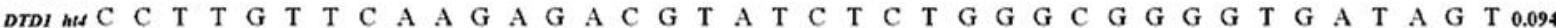

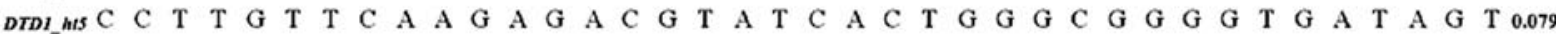
olhers 0.063

C

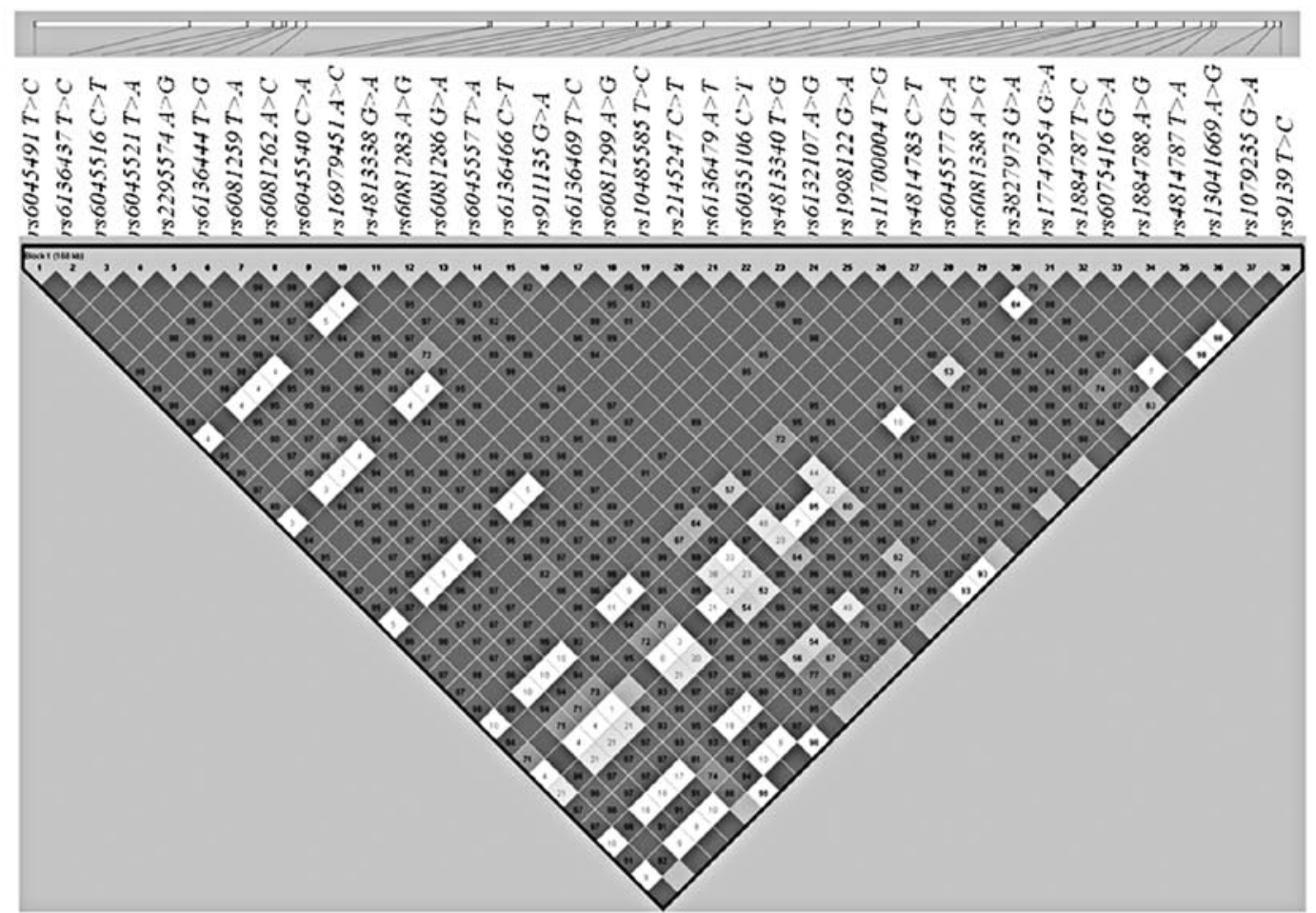

Figure 1. Physical map, haplotypes, and linkage disequilibrium of the DTD1 gene. (A) Schematic gene map and SNPs in the DTD1 gene on chromosome 20 p11.23 (176 kb). Black blocks represent coding exons and white blocks represent 5' and 3' UTRs. The first base of translation site was denoted as nucleotide +1 . SNPs in absolute linkage are indicated by brackets $\left(\mathrm{r}^{2}=1\right)$. (B) Haplotypes of DTD1. (C) LD coefficient (ID') among DTD1 SNPs in a Korean population. UTR, untranslated region. 
Table II. Genotype and allele frequency distribution of DTD1 variants.

\begin{tabular}{|c|c|c|c|c|c|}
\hline Loci & Position & Allele & MAF & Heterozygosity & HWE \\
\hline rs6045491 & Intron 2 & $\mathrm{~T}>\mathrm{C}$ & 0.323 & 0.437 & 0.258 \\
\hline$r s 6136437$ & Intron 3 & $\mathrm{~T}>\mathrm{C}$ & 0.180 & 0.295 & 0.253 \\
\hline$r s 6045516$ & Intron 3 & $\mathrm{C}>\mathrm{T}$ & 0.323 & 0.437 & 0.258 \\
\hline$r s 6045521$ & Intron 3 & $\mathrm{~T}>\mathrm{A}$ & 0.323 & 0.437 & 0.258 \\
\hline$r s 2295574$ & Intron 4 & $\mathrm{~A}>\mathrm{G}$ & 0.322 & 0.436 & 0.223 \\
\hline rs6136444 & Intron 4 & $\mathrm{~T}>\mathrm{G}$ & 0.105 & 0.189 & 0.407 \\
\hline rs6081259 & Intron 4 & $\mathrm{~T}>\mathrm{A}$ & 0.303 & 0.422 & 0.945 \\
\hline$r s 6081262$ & Intron 4 & $\mathrm{~A}>\mathrm{C}$ & 0.323 & 0.438 & 0.170 \\
\hline rs6045540 & Intron 4 & $\mathrm{C}>\mathrm{A}$ & 0.327 & 0.440 & 0.196 \\
\hline rs16979451 & Intron 4 & $\mathrm{~A}>\mathrm{C}$ & 0.314 & 0.431 & 0.904 \\
\hline$r s 4813338$ & Intron 4 & $\mathrm{G}>\mathrm{A}$ & 0.459 & 0.497 & 0.464 \\
\hline$r s 6081283$ & Intron 4 & $A>G$ & 0.146 & 0.250 & 0.905 \\
\hline$r s 6081286$ & Intron 4 & $\mathrm{G}>\mathrm{A}$ & 0.150 & 0.255 & 0.782 \\
\hline$r s 6045557$ & Intron 4 & $\mathrm{~T}>\mathrm{A}$ & 0.184 & 0.300 & 0.271 \\
\hline$r s 6136466$ & Intron 4 & $\mathrm{C}>\mathrm{T}$ & 0.155 & 0.262 & 0.773 \\
\hline rs911135 & Intron 4 & $\mathrm{G}>\mathrm{A}$ & 0.441 & 0.493 & 0.550 \\
\hline$r s 6136469$ & Intron 4 & $\mathrm{~T}>\mathrm{C}$ & 0.124 & 0.217 & 0.185 \\
\hline rs6081299 & Intron 4 & $\mathrm{~A}>\mathrm{G}$ & 0.142 & 0.243 & 0.861 \\
\hline rs10485585 & Intron 4 & $\mathrm{~T}>\mathrm{C}$ & 0.300 & 0.420 & 0.726 \\
\hline$r s 2145247$ & Intron 4 & $\mathrm{C}>\mathrm{T}$ & 0.299 & 0.419 & 0.696 \\
\hline$r s 6136479$ & Intron 4 & $\mathrm{~A}>\mathrm{T}$ & 0.099 & 0.178 & 0.338 \\
\hline rs6035106 & Intron 4 & $\mathrm{C}>\mathrm{T}$ & 0.459 & 0.497 & 0.417 \\
\hline$r s 4813340$ & Intron 4 & $\mathrm{~T}>\mathrm{G}$ & 0.321 & 0.436 & 0.590 \\
\hline rs6132107 & Intron 4 & $A>G$ & 0.181 & 0.296 & 0.235 \\
\hline rs1998122 & Intron 4 & $\mathrm{G}>\mathrm{A}$ & 0.377 & 0.470 & 0.420 \\
\hline rs11700004 & Intron 4 & $\mathrm{~T}>\mathrm{G}$ & 0.181 & 0.296 & 0.235 \\
\hline$r s 4814783$ & Intron 4 & $\mathrm{C}>\mathrm{T}$ & 0.324 & 0.438 & 0.943 \\
\hline rs6045577 & Intron 4 & $\mathrm{G}>\mathrm{A}$ & 0.420 & 0.487 & 0.869 \\
\hline$r s 6081338$ & Intron 4 & $\mathrm{~A}>\mathrm{G}$ & 0.123 & 0.216 & 0.198 \\
\hline rs3827973 & Intron 5 & $\mathrm{G}>\mathrm{A}$ & 0.346 & 0.452 & 0.877 \\
\hline rs17747954 & Intron 5 & $\mathrm{G}>\mathrm{A}$ & 0.055 & 0.104 & 0.940 \\
\hline rs1884787 & Intron 5 & $\mathrm{~T}>\mathrm{C}$ & 0.433 & 0.491 & 0.589 \\
\hline$r s 6075416$ & Intron 5 & $\mathrm{G}>\mathrm{A}$ & 0.302 & 0.422 & 0.859 \\
\hline$r s 1884788$ & Intron 5 & $\mathrm{~A}>\mathrm{G}$ & 0.302 & 0.422 & 0.859 \\
\hline$r s 4814787$ & Intron 5 & $\mathrm{~T}>\mathrm{A}$ & 0.419 & 0.487 & 0.614 \\
\hline rs13041669 & Intron 5 & $\mathrm{~A}>\mathrm{G}$ & 0.316 & 0.432 & 0.769 \\
\hline rs1079235 & Intron 5 & $\mathrm{G}>\mathrm{A}$ & 0.454 & 0.496 & 0.270 \\
\hline$r s 9139$ & Exon 6 & $\mathrm{~T}>\mathrm{C}$ & 0.036 & 0.069 & 0.351 \\
\hline DTD1_ht1 & & & 0.266 & 0.390 & 0.578 \\
\hline$D T D 1 \_h t 2$ & & & 0.265 & 0.390 & 0.307 \\
\hline DTD1_ht3 & & & 0.093 & 0.168 & 0.259 \\
\hline DTD1_ht4 & & & 0.093 & 0.169 & 0.489 \\
\hline DTD1_ht5 & & & 0.076 & 0.140 & 0.361 \\
\hline
\end{tabular}

MAF, minor allele frequency; HWE, Hardy-Weinberg equilibrium.

aspirin-intolerance is manifested through severe acute asthma attacks requiring hospital admission and mechanical ventilation to support the failing lungs $(7,8)$. Once developed,
AIA cases are considered fatal despite treatment with fastacting medicines and avoidance of aspirin. As a precipitating factor in life-threatening asthma attacks, researchers are 
Table III. Association analysis of DTD1 polymorphisms and haplotypes with AIA.

\begin{tabular}{|c|c|c|c|c|c|c|c|c|c|c|}
\hline \multirow[b]{2}{*}{ Loci } & \multirow[b]{2}{*}{ Position } & \multicolumn{2}{|c|}{ MAF } & \multicolumn{2}{|c|}{ Co-dominant } & \multicolumn{2}{|c|}{ Dominant } & \multicolumn{3}{|c|}{ Recessive } \\
\hline & & $\begin{array}{c}\text { AIA } \\
(n=163)\end{array}$ & $\begin{array}{c}\text { ATA } \\
(\mathrm{n}=429)\end{array}$ & OR $(95 \% \mathrm{CI})$ & P-value ${ }^{a}$ & OR $(95 \% \mathrm{CI})$ & P-value ${ }^{a}$ & & $\mathrm{R}(95 \% \mathrm{CI})$ & P-value \\
\hline rs6045491 & Intron 2 & 0.313 & 0.321 & $1.00(0.75-1.33)$ & 0.98 & $1.04(0.72-1.51)$ & 0.82 & 0.85 & $(0.43-1.68)$ & 0.64 \\
\hline rs6136437 & Intron 3 & 0.181 & 0.179 & $1.04(0.74-1.47)$ & 0.82 & $1.14(0.77-1.68)$ & 0.52 & 0.42 & $(0.09-1.89)$ & 0.26 \\
\hline$r s 6045516$ & Intron 3 & 0.313 & 0.321 & $1.00(0.75-1.33)$ & 0.98 & $1.04(0.72-1.51)$ & 0.82 & 0.85 & $(0.43-1.68)$ & 0.64 \\
\hline$r s 6045521$ & Intron 3 & 0.313 & 0.321 & $1.00(0.75-1.33)$ & 0.98 & $1.04(0.72-1.51)$ & 0.82 & 0.85 & $(0.43-1.68)$ & 0.64 \\
\hline$r s 2295574$ & Intron 4 & 0.313 & 0.319 & $1.00(0.75-1.34)$ & 1.00 & $1.05(0.72-1.52)$ & 0.81 & 0.86 & $(0.43-1.71)$ & 0.67 \\
\hline rs6136444 & Intron 4 & 0.107 & 0.105 & $1.07(0.70-1.63)$ & 0.77 & $0.98(0.62-1.56)$ & 0.95 & 3.58 & $(0.69-18.51)$ & 0.13 \\
\hline$r s 6081259$ & Intron 4 & 0.282 & 0.308 & $0.88(0.66-1.18)$ & 0.40 & $0.93(0.64-1.34)$ & 0.68 & 0.65 & $(0.32-1.32)$ & 0.24 \\
\hline$r s 6081262$ & Intron 4 & 0.313 & 0.322 & $0.99(0.74-1.32)$ & 0.93 & $1.02(0.71-1.48)$ & 0.91 & 0.86 & $(0.44-1.71)$ & 0.67 \\
\hline$r s 6045540$ & Intron 4 & 0.316 & 0.326 & $0.98(0.73-1.31)$ & 0.88 & $1.03(0.71-1.49)$ & 0.90 & 0.82 & $(0.42-1.63)$ & 0.58 \\
\hline rs16979451 & Intron 4 & 0.293 & 0.317 & $0.90(0.68-1.20)$ & 0.47 & $0.95(0.66-1.38)$ & 0.80 & 0.67 & $(0.34-1.32)$ & 0.24 \\
\hline$r s 4813338$ & Intron 4 & 0.426 & 0.461 & $0.88(0.67-1.14)$ & 0.33 & $0.91(0.61-1.36)$ & 0.63 & 0.75 & $(0.46-1.22)$ & 0.25 \\
\hline rs6081283 & Intron 4 & 0.135 & 0.145 & $0.94(0.64-1.38)$ & 0.75 & $0.91(0.60-1.39)$ & 0.67 & 1.15 & $(0.30-4.38)$ & 0.84 \\
\hline$r s 6081286$ & Intron 4 & 0.138 & 0.149 & $0.93(0.64-1.35)$ & 0.71 & $0.89(0.58-1.35)$ & 0.58 & 1.29 & $(0.39-4.25)$ & 0.68 \\
\hline rs6045557 & Intron 4 & 0.184 & 0.184 & $1.03(0.73-1.46)$ & 0.86 & $1.13(0.77-1.66)$ & 0.54 & 0.40 & $(0.09-1.81)$ & 0.23 \\
\hline rs6136466 & Intron 4 & 0.138 & 0.155 & $0.89(0.61-1.29)$ & 0.53 & $0.84(0.55-1.27)$ & 0.40 & 1.29 & $(0.40-4.18)$ & 0.67 \\
\hline$r s 911135$ & Intron 4 & 0.399 & 0.446 & $0.81(0.62-1.06)$ & 0.13 & $0.83(0.56-1.23)$ & 0.35 & 0.66 & $(0.39-1.10)$ & 0.11 \\
\hline$r s 6136469$ & Intron 4 & 0.123 & 0.121 & $1.00(0.67-1.50)$ & 1.00 & $0.94(0.60-1.45)$ & 0.77 & 2.53 & $(0.55-11.74)$ & 0.23 \\
\hline rs6081299 & Intron 4 & 0.126 & 0.141 & $0.86(0.58-1.27)$ & 0.45 & $0.82(0.53-1.26)$ & 0.36 & 1.24 & $(0.32-4.80)$ & 0.76 \\
\hline rs 10485585 & Intron 4 & 0.276 & 0.304 & $0.86(0.65-1.15)$ & 0.32 & $0.89(0.62-1.29)$ & 0.55 & 0.65 & $(0.32-1.31)$ & 0.23 \\
\hline$r s 2145247$ & Intron 4 & 0.276 & 0.304 & $0.86(0.65-1.15)$ & 0.32 & $0.90(0.62-1.30)$ & 0.56 & 0.64 & $(0.32-1.30)$ & 0.22 \\
\hline rs6136479 & Intron 4 & 0.110 & 0.094 & $1.21(0.79-1.88)$ & 0.38 & $1.25(0.79-1.98)$ & 0.34 & 0.86 & $(0.09-8.51)$ & 0.89 \\
\hline rs6035106 & Intron 4 & 0.414 & 0.465 & $0.82(0.62-1.07)$ & 0.14 & $0.87(0.58-1.30)$ & 0.48 & 0.64 & $(0.39-1.05)$ & 0.08 \\
\hline$r s 4813340$ & Intron 4 & 0.367 & 0.311 & $1.24(0.94-1.64)$ & 0.12 & $1.21(0.83-1.76)$ & 0.32 & 1.64 & $(0.94-2.86)$ & 0.08 \\
\hline rs6132107 & Intron 4 & 0.184 & 0.179 & $1.06(0.75-1.49)$ & 0.76 & $1.16(0.78-1.70)$ & 0.47 & 0.42 & $(0.09-1.89)$ & 0.26 \\
\hline rs1998122 & Intron 4 & 0.420 & 0.371 & $1.20(0.91-1.58)$ & 0.19 & $1.17(0.79-1.73)$ & 0.43 & 1.45 & $(0.87-2.42)$ & 0.15 \\
\hline rs11700004 & Intron 4 & 0.184 & 0.179 & $1.06(0.75-1.49)$ & 0.76 & $1.16(0.78-1.70)$ & 0.47 & 0.42 & $(0.09-1.89)$ & 0.26 \\
\hline$r s 4814783$ & Intron 4 & 0.367 & 0.315 & $1.22(0.93-1.61)$ & 0.15 & $1.16(0.79-1.68)$ & 0.45 & 1.69 & $(0.97-2.95)$ & 0.07 \\
\hline rs6045577 & Intron 4 & 0.380 & 0.426 & $0.82(0.63-1.07)$ & 0.15 & $0.84(0.57-1.23)$ & 0.37 & 0.66 & $(0.39-1.12)$ & 0.12 \\
\hline rs6081338 & Intron 4 & 0.123 & 0.120 & $1.01(0.67-1.52)$ & 0.96 & $0.95(0.61-1.47)$ & 0.81 & 2.53 & $(0.55-11.74)$ & 0.23 \\
\hline rs3827973 & Intron 5 & 0.368 & 0.340 & $1.12(0.85-1.47)$ & 0.43 & $1.24(0.85-1.80)$ & 0.27 & 0.99 & $(0.55-1.75)$ & 0.96 \\
\hline rs177477954 & Intron 5 & 0.034 & 0.063 & $0.54(0.28-1.07)$ & 0.08 & $0.55(0.27-1.09)$ & 0.08 & & - & 0.98 \\
\hline rs1884787 & Intron 5 & 0.408 & 0.436 & $0.91(0.69-1.19)$ & 0.48 & $0.99(0.67-1.47)$ & 0.95 & 0.73 & $(0.44-1.21)$ & 0.22 \\
\hline$r s 6075416$ & Intron 5 & 0.279 & 0.308 & $0.88(0.66-1.17)$ & 0.38 & $0.91(0.63-1.32)$ & 0.61 & 0.67 & $(0.33-1.35)$ & 0.26 \\
\hline rs 1884788 & Intron 5 & 0.279 & 0.308 & $0.88(0.66-1.17)$ & 0.38 & $0.91(0.63-1.32)$ & 0.61 & 0.67 & $(0.33-1.35)$ & 0.26 \\
\hline rs4814787 & Intron 5 & 0.396 & 0.422 & $0.90(0.69-1.17)$ & 0.43 & $0.95(0.64-1.40)$ & 0.78 & 0.75 & $(0.45-1.25)$ & 0.27 \\
\hline$r s 13041669$ & Intron 5 & 0.359 & 0.305 & $1.23(0.93-1.62)$ & 0.14 & $1.20(0.82-1.74)$ & 0.35 & 1.60 & $(0.91-2.82)$ & 0.10 \\
\hline rs1079235 & Intron 5 & 0.414 & 0.459 & $0.85(0.64-1.11)$ & 0.22 & $0.89(0.60-1.34)$ & 0.58 & 0.68 & $(0.41-1.13)$ & 0.13 \\
\hline$r s 9139$ & Exon 6 & 0.028 & 0.042 & $0.68(0.32-1.46)$ & 0.32 & $0.68(0.32-1.46)$ & 0.32 & & - & - \\
\hline DTD1_ht1 & & 0.224 & 0.277 & $0.76(0.56-1.03)$ & 0.08 & $0.74(0.51-1.08)$ & 0.12 & 0.58 & $(0.26-1.30)$ & 0.18 \\
\hline DTD1_ht2 & & 0.294 & 0.256 & $1.16(0.87-1.54)$ & 0.32 & $1.10(0.76-1.60)$ & 0.61 & 1.57 & $(0.82-2.97)$ & 0.17 \\
\hline DTD1_ht3 & & 0.083 & 0.094 & $0.87(0.54-1.41)$ & 0.58 & $0.84(0.51-1.38)$ & 0.49 & 2.20 & $(0.19-24.96)$ & 0.52 \\
\hline DTD1_ht4 & & 0.101 & 0.090 & $1.17(0.75-1.83)$ & 0.49 & $1.20(0.75-1.93)$ & 0.45 & 0.86 & $(0.09-8.51)$ & 0.89 \\
\hline DTD1_ht5 & & 0.067 & 0.080 & $0.83(0.49-1.40)$ & 0.48 & $0.84(0.50-1.44)$ & 0.53 & & - & 0.98 \\
\hline
\end{tabular}

AIA, aspirin-intolerant asthma; ATA, aspirin tolerant asthma; OR, odds ratio; CI, confidence interval. ${ }^{\text {a }}<0.05$. Co-dominant, dominant and recessive models of logistic regression analyses were used to calculate ORs and the $95 \%$ CIs controlling for age, gender, smoking status, atopy and BMI as covariates. 
Table IV. Regression analysis of DTD1 polymorphisms and haplotypes with fall rate of $\mathrm{FEV}_{1}$ by aspirin provocation.

\begin{tabular}{|c|c|c|c|c|c|c|c|c|c|c|}
\hline Loci & $\mathrm{C} / \mathrm{C}$ & & $\mathrm{C} / \mathrm{R}$ & $\mathrm{R} / \mathrm{R}$ & $\mathrm{Pa}$ & $\mathrm{Pa}^{\text {corr }}$ & $\mathrm{Pb}$ & $\mathrm{Pb}^{\text {corr }}$ & $\mathrm{Pc}$ & $\mathrm{Pc}^{\text {corr }}$ \\
\hline rs6045491 & $270(9.37 \pm 12.93)$ & 265 & $(9.32 \pm 13.59)$ & $57 \quad(8.19 \pm 13.01)$ & 0.77 & - & 0.86 & - & 0.74 & - \\
\hline$r s 6136437$ & $395(9.21 \pm 13.02)$ & 181 & $(9.42 \pm 13.90)$ & $(7.59 \pm 10.43)$ & 0.96 & - & 0.94 & - & 0.7 & - \\
\hline$r s 6045516$ & $270(9.37 \pm 12.93)$ & 265 & $(9.32 \pm 13.59)$ & $(8.19 \pm 13.01)$ & 0.77 & - & 0.86 & - & 0.74 & - \\
\hline rs6045521 & $270(9.37 \pm 12.93)$ & 265 & $(9.32 \pm 13.59)$ & $57 \quad(8.19 \pm 13.01)$ & 0.77 & - & 0.86 & - & 0.74 & - \\
\hline$r s 2295574$ & $270(9.37 \pm 12.93)$ & 265 & $(9.32 \pm 13.59)$ & $56 \quad(8.32 \pm 13.09)$ & 0.8 & - & 0.87 & - & 0.77 & - \\
\hline$r s 6136444$ & $471(9.39 \pm 13.23)$ & 115 & $(7.91 \pm 12.09)$ & $6(22.25 \pm 25.23)$ & 0.85 & - & 0.66 & - & 0.01 & NS \\
\hline$r s 6081259$ & $289(9.11 \pm 13.01)$ & 251 & $(9.76 \pm 13.85)$ & $52 \quad(7.37 \pm 11.15)$ & 0.79 & - & 0.76 & - & 0.24 & - \\
\hline$r s 6081262$ & $268(9.38 \pm 12.97)$ & 268 & $(9.28 \pm 13.53)$ & $56 \quad(8.32 \pm 13.09)$ & 0.78 & - & 0.85 & - & 0.78 & - \\
\hline rs6045540 & $265(9.33 \pm 12.98)$ & 269 & $(9.37 \pm 13.56)$ & $58 \quad(8.16 \pm 12.90)$ & 0.78 & - & 0.89 & - & 0.7 & - \\
\hline rs16979451 & $280(8.99 \pm 12.91)$ & 253 & $(9.93 \pm 14.06)$ & $56 \quad(7.28 \pm 10.89)$ & 0.9 & - & 0.57 & - & 0.22 & - \\
\hline$r s 4813338$ & $173(9.15 \pm 13.19)$ & 300 & $(9.72 \pm 13.31)$ & $117 \quad(8.18 \pm 13.16)$ & 0.74 & - & 0.73 & - & 0.33 & - \\
\hline$r s 6081283$ & $434(9.49 \pm 13.38)$ & 146 & $(8.18 \pm 12.07)$ & $12(12.84 \pm 19.79)$ & 0.76 & - & 0.49 & - & 0.26 & - \\
\hline$r s 6081286$ & $431(9.52 \pm 13.42)$ & 147 & $(8.08 \pm 12.01)$ & $14(12.49 \pm 18.37)$ & 0.73 & - & 0.45 & - & 0.29 & - \\
\hline rs6045557 & $391(9.22 \pm 13.07)$ & 184 & $(9.41 \pm 13.82)$ & $17 \quad(7.62 \pm 10.10)$ & 0.94 & - & 0.98 & - & 0.74 & - \\
\hline$r s 6136466$ & $426(9.59 \pm 13.48)$ & 150 & $(8.01 \pm 11.94)$ & $15(11.79 \pm 17.77)$ & 0.59 & - & 0.34 & - & 0.34 & - \\
\hline$r s 911135$ & $186(9.50 \pm 13.31)$ & 298 & $(9.53 \pm 13.32)$ & $108 \quad(7.95 \pm 12.81)$ & 0.44 & - & 0.86 & - & 0.24 & - \\
\hline$r s 6136469$ & $453(9.46 \pm 13.46)$ & 132 & $(7.88 \pm 11.36)$ & $7(19.97 \pm 23.81)$ & 0.86 & - & 0.44 & - & 0.02 & NS \\
\hline rs6081299 & $438(9.60 \pm 13.49)$ & 142 & $(7.79 \pm 11.57)$ & $11(13.92 \pm 20.38)$ & 0.52 & - & 0.27 & - & 0.2 & - \\
\hline rs 10485585 & $294(9.11 \pm 12.92)$ & 246 & $(9.71 \pm 13.99)$ & $52 \quad(7.68 \pm 11.08)$ & 0.85 & - & 0.77 & - & 0.34 & - \\
\hline$r s 2145247$ & $294(9.11 \pm 12.92)$ & 245 & $(9.73 \pm 14.01)$ & $52 \quad(7.60 \pm 11.09)$ & 0.84 & - & 0.77 & - & 0.32 & - \\
\hline$r s 6136479$ & $477(8.72 \pm 12.62)$ & 111 & $(11.59 \pm 15.50)$ & $(5.58 \pm 9.14)$ & 0.09 & - & 0.06 & - & 0.62 & - \\
\hline rs6035106 & $173(9.22 \pm 13.21)$ & 302 & $(9.84 \pm 13.52)$ & $117 \quad(7.68 \pm 12.42)$ & 0.52 & - & 0.8 & - & 0.16 & - \\
\hline$r s 4813340$ & $267(8.60 \pm 12.78)$ & 248 & $(9.60 \pm 13.61)$ & $65(10.75 \pm 13.67)$ & 0.26 & - & 0.34 & - & 0.36 & - \\
\hline$r s 6132107$ & $394(9.21 \pm 13.02)$ & 182 & $(9.42 \pm 13.90)$ & $16 \quad(7.59 \pm 10.43)$ & 0.92 & - & 0.98 & - & 0.7 & - \\
\hline rs1998122 & $220(8.58 \pm 13.26)$ & 288 & $(9.48 \pm 13.17)$ & $83(10.18 \pm 13.41)$ & 0.39 & - & 0.48 & - & 0.49 & - \\
\hline rs11700004 & $394(9.21 \pm 13.02)$ & 182 & $(9.42 \pm 13.90)$ & $16 \quad(7.59 \pm 10.43)$ & 0.92 & - & 0.98 & - & 0.7 & - \\
\hline$r s 4814783$ & $268(8.60 \pm 12.75)$ & 258 & $(9.46 \pm 13.60)$ & $65(10.75 \pm 13.67)$ & 0.28 & - & 0.39 & - & 0.34 & - \\
\hline rs6045577 & $202(9.12 \pm 13.06)$ & 287 & $(9.70 \pm 13.42)$ & $101 \quad(8.22 \pm 13.12)$ & 0.78 & - & 0.73 & - & 0.34 & - \\
\hline$r s 6081338$ & $454(9.45 \pm 13.45)$ & 131 & $(7.91 \pm 11.40)$ & $7(19.97 \pm 23.81)$ & 0.87 & - & 0.46 & - & 0.02 & NS \\
\hline rs3827973 & $252(8.32 \pm 12.52)$ & 269 & $(10.27 \pm 13.95)$ & $71 \quad(8.54 \pm 12.67)$ & 0.45 & - & 0.18 & - & 0.65 & - \\
\hline rs 17747954 & $529(9.51 \pm 13.48)$ & 61 & $(7.05 \pm 10.67)$ & $2 \quad(1.70 \pm 0.99)$ & 0.12 & - & 0.13 & - & 0.55 & - \\
\hline rs1884787 & $190(9.06 \pm 13.10)$ & 296 & $(9.83 \pm 13.72)$ & $106 \quad(7.86 \pm 11.95)$ & 0.73 & - & 0.64 & - & 0.23 & - \\
\hline$r s 6075416$ & $290(9.17 \pm 13.03)$ & 250 & $(9.62 \pm 13.86)$ & $52 \quad(7.69 \pm 11.07)$ & 0.82 & - & 0.84 & - & 0.38 & - \\
\hline$r s 1884788$ & $290(9.17 \pm 13.03)$ & 250 & $(9.62 \pm 13.86)$ & $52 \quad(7.69 \pm 11.07)$ & 0.82 & - & 0.84 & - & 0.38 & - \\
\hline$r s 4814787$ & $199(9.22 \pm 13.21)$ & 294 & $(9.62 \pm 13.56)$ & $99 \quad(8.12 \pm 12.23)$ & 0.69 & - & 0.84 & - & 0.33 & - \\
\hline rs13041669 & $276(8.48 \pm 12.48)$ & 252 & $(9.69 \pm 13.90)$ & $63(10.72 \pm 13.69)$ & 0.24 & - & 0.3 & - & 0.4 & - \\
\hline rs1079235 & $174(9.07 \pm 13.04)$ & 306 & $(9.94 \pm 13.81)$ & $112 \quad(7.56 \pm 11.71)$ & 0.61 & - & 0.63 & - & 0.15 & - \\
\hline$r s 9139$ & $546(9.39 \pm 13.43)$ & 45 & $(7.34 \pm 10.43)$ & - & 0.3 & - & 0.3 & - & - & - \\
\hline DTD1_ht1 & $324(9.31 \pm 12.93)$ & 226 & $(9.59 \pm 13.92)$ & $42 \quad(6.72 \pm 11.46)$ & 0.56 & - & 0.95 & - & 0.2 & - \\
\hline DTD1_ht2 & $324(8.58 \pm 12.39)$ & 220 & $(10.09 \pm 14.33)$ & $48 \quad(9.71 \pm 13.37)$ & 0.34 & - & 0.28 & - & 0.79 & - \\
\hline DTD1_ht3 & $485(9.57 \pm 13.42)$ & 104 & $(7.41 \pm 11.79)$ & $3(17.83 \pm 23.69)$ & 0.33 & - & 0.21 & - & 0.2 & - \\
\hline DTD1_ht4 & $484(8.78 \pm 12.59)$ & 104 & $(11.50 \pm 15.80)$ & $(5.58 \pm 9.14)$ & 0.1 & - & 0.07 & - & 0.62 & - \\
\hline DTD1_ht5 & $506(9.73 \pm 13.63)$ & 84 & $(6.48 \pm 10.14)$ & $(-0.10 \pm 6.93)$ & 0.02 & NS & 0.02 & NS & 0.32 & - \\
\hline
\end{tabular}

Co-dominant, dominant and recessive models of multiple linear regression analyses were performed controlling for age, gender, smoking status, atopy and $\mathrm{BMI}$ as covariates. $\mathrm{C} / \mathrm{C}, \mathrm{C} / \mathrm{R}$ and $\mathrm{R} / \mathrm{R}$ indicate the homozygotes of the common allele, and the heterozygotes and homozygotes of the rare allele, respectively. Bold values indicate $\mathrm{P}<0.05$. $\mathrm{Pa}, \mathrm{Pb}$ and $\mathrm{Pc}$ refer to $\mathrm{P}$-values of the co-dominant, dominant and recessive models, respectively. $\mathrm{Pa}^{\text {corr }}, \mathrm{Pb}^{\text {corr }}$ and $\mathrm{Pc}^{\text {corr }}$ refer to $\mathrm{P}$-values after multiple testing corrections (29.1223). NS, not significant. 
now actively engaged in identifying mechanisms involved in the development of AIA that may lead to novel targets for treatment of the disease. Despite previous reports on genetic markers of bronchial hypersensitivity as a result of aspirin intake, the exact mechanisms of the disease are still unclear and susceptibility genes still need to be identified. Findings from this study may contribute to the current knowledge of AIA pathogenesis.

Although D-amino acids are thought to not be incorporated in proteins, several aminoacyl-tRNA synthetases (aaRSs) such as the tyrRS are capable of transferring the D-isomer of their amino acid onto their cognate tRNA, resulting in accumulation of the metabolically inactive D-aminoacyl-tRNAs (21). The DTDI gene counters this process and interacts with tyrRS by possessing a D-tyrosyl-tRNA deacylase that cleaves the ester bond between a tRNA molecule and a D-amino acid (21). Misregulation of DTDI gene expression may increase the toxicity of D-amino acids such as D-tryptophan, an enzyme that has been implicated in AIA etiology by producing kynurenine and serotonin (18-20). Furthermore, Wakasugi and Schimmel have revealed that cytoplasmic tyrRS has proinflammatory cytokine functions similar to interleukin- 8 (IL-8) and to the endothelial monocyte-activating polypeptide II (EMAP II) (33). Previous studies have demonstrated the association between an IL-8 polymorphism and respiratory syncytial virus (RSV) bronchiolitis, a condition that may progress to bronchial asthma $(34,35)$. In addition, IL-8 has been implicated in neutrophilic inflammation associated with severe asthma $(36,37)$, whereas EMAP II has been known to stimulate the production of tumor necrosis factor- $\alpha(\mathrm{TNF} \alpha)$ (38), an AIA-susceptibility gene (39). Given the indirect role of DTDI in AIA susceptibility by interacting with tyrRS and catalyzing the formation of D-amino acids, we investigated the association of DTDI genetic variants with the risk of AIA in a Korean population.

In this study, differences in the distributions of 38 genotyped SNPs and 5 major haplotypes in DTDl were analyzed for a possible association with the risk of AIA and decline rate of $\mathrm{FEV}_{1}$. Results from the logistic regression analysis revealed a lack of association between $D T D 1$ variants and AIA susceptibility. Furthermore, since the decline rate of $\mathrm{FEV}_{1}$ is a crucial diagnostic marker for AIA, further analysis was performed. Our initial findings revealed associations of several DTD1 SNPs (rs6136444, rs6136469 and rs6081338) and of one haplotype (DTDI_ht5) with the decline rate of $\mathrm{FEV}_{1}$ induced by aspirin provocation. Patients with the rare allele $(\mathrm{R} / \mathrm{R}$ genotype) of the polymorphisms were in greater risk of developing AIA compared to subjects having other genotypes $(\mathrm{C} / \mathrm{C}$ and $\mathrm{C} / \mathrm{R})$. However, the significant signals were not retained after multiple testing corrections, suggesting that $D T D 1$ polymorphisms do not affect pulmonary function abnormalities in AIA patients.

Although the exact molecular mechanisms are not clear, the $D T D 1$ gene can counteract the accumulation of inactive tRNA molecules (14) and promote defense mechanisms against the harmful effects of D-amino acids including tryptophan, an enzyme that is down-regulated by aspirin (40) and has been implicated in AIA susceptibility in several studies (18-20). The small sample size $(n=592)$ may serve as a potential limitation to this preliminary study, and therefore, in order to clarify the relationship between $D T D I$ variants and the risk of AIA with high statistical power, further replication studies should use larger scale samples in various ethnic groups $(\mathrm{n}>1,000)$.

To our knowledge, this study is the first to explore the relationship between $D T D 1$ gene variations and AIA pathogenesis. To conclude, our findings provide evidence that $D T D 1$ variants do not influence AIA and the decline rate of $\mathrm{FEV}_{1}$ in a Korean population. However, considering the important function of DTD1 in AIA pathogenesis, future replication studies in a larger cohort are recommended. These studies may be useful in the current genetic etiology of AIA susceptibility.

\section{Acknowledgements}

This study was supported by the Korea Science and Engineering Foundation (KOSEF) funded by the Korea government (MEST) (nos. 2009-0080157 and 2010-0011206). This study was supported by a grant of the Korea Healthcare Technology R\&D Project, Ministry for Health, Welfare and Family Affairs, Republic of Korea (A010249). The DNA samples were generously provided by the Soonchunhyang University, Bucheon Hospital Biobank, a member of the National Biobank of Korea, supported by the Ministry of Health, Welfare and Family Affairs, Republic of Korea.

\section{References}

1. Bel EH: Clinical phenotypes of asthma. Curr Opin Pulm Med 10: 44-50, 2004.

2. Babu KS and Salvi SS: Aspirin and asthma. Chest 118: 1470-1476, 2000.

3. Sturtevant J: NSAID-induced bronchospasm: a common and serious problem. A report from MEDSAFE, the New Zealand Medicines and Medical Devices Safety Authority. NZ Dent J 95: 84, 1999.

4. Spector SL, Wangaard CH and Farr RS: Aspirin and concomitant idiosyncrasies in adult asthmatic patients. J Allergy Clin Immunol 64: 500-506, 1979.

5. Samter M and Beers RF Jr: Intolerance to aspirin. Clinical studies and consideration of its pathogenesis. Ann Intern Med 68: 975-983, 1968.

6. Stevenson DD: Diagnosis, prevention, and treatment of adverse reactions to aspirin and nonsteroidal anti-inflammatory drugs. J Allergy Clin Immunol 74: 617-622, 1984.

7. Marquette $\mathrm{CH}$, Saulnier F, Leroy $\mathrm{O}$, et al: Long-term prognosis of near-fatal asthma. A 6-year follow-up study of 145 asthmatic patients who underwent mechanical ventilation for a near-fatal attack of asthma. Am Rev Respir Dis 146: 76-81, 1992.

8. Picado C, Castillo JA, Montserrat JM and Agusti-Vidal A: Aspirin-intolerance as a precipitating factor of life-threatening attacks of asthma requiring mechanical ventilation. Eur Respir J 2: 127-129, 1989.

9. Pasaje CF, Kim JH, Park BL, et al: Association of SLC6A12 variants with aspirin-intolerant asthma in a Korean population. Ann Hum Genet 74: 326-334, 2010.

10. Kim JY, Kim JH, Park TJ, et al: Positive association between aspirin-intolerant asthma and genetic polymorphisms of FSIP1: a case-case study. BMC Pulm Med 10: 34, 2010.

11. Pasaje CF, Kim JH, Park BL, et al: A possible association of EMID2 polymorphisms with aspirin hypersensitivity in asthma. Immunogenetics 63: 13-21, 2011.

12. Meng X, Chen J, Yang Q, et al: Cloning and identification of a novel cDNA which may be associated with FKBP25. Biochem Genet 40: 303-310, 2002.

13. Soutourina J, Plateau P and Blanquet S: Metabolism of D-aminoacyl-tRNAs in Escherichia coli and Saccharomyces cerevisiae cells. J Biol Chem 275: 32535-32542, 2000.

14. Soutourina J, Plateau P, Delort F, Peirotes A and Blanquet S: Functional characterization of the D-Tyr-tRNATyr deacylase from Escherichia coli. J Biol Chem 274: 19109-19114, 1999. 
15. Wirleitner B, Neurauter G, Schrocksnadel K, Frick B and Fuchs D: Interferon-gamma-induced conversion of tryptophan: immunologic and neuropsychiatric aspects. Curr Med Chem 10: $1581-1591,2003$

16. Munn DH, Shafizadeh E, Attwood JT, Bondarev I, Pashine A and Mellor AL: Inhibition of T cell proliferation by macrophage tryptophan catabolism. J Exp Med 189: 1363-1372, 1999.

17. Seroogy CM and Gern JE: The role of T regulatory cells in asthma. J Allergy Clin Immunol 116: 996-999, 2005.

18. Collipp PJ, Chen SY, Sharma RK, Balachandar V and Maddaiah VT: Tryptophane metabolism in bronchial asthma. Ann Allergy 35: 153-158, 1975.

19. Warraki SE, el-Gammal MY, el-Asmar MF and Wahba N: Serum kynurenine in bronchial asthma and chronic bronchitis. Chest 57: 148-150, 1970.

20. Lechin F, van der Dijs B, Orozco B, Lechin M and Lechin AE: Increased levels of free serotonin in plasma of symptomatic asthmatic patients. Ann Allergy Asthma Immunol 77: 245-253, 1996.

21. Soutourina O, Soutourina J, Blanquet S and Plateau P: Formation of D-tyrosyl-tRNATyr accounts for the toxicity of D-tyrosine toward Escherichia coli. J Biol Chem 279: 42560-42565, 2004.

22. Global Strategy for Asthma Management and Prevention. Global Initiative for Asthma (GINA), 1993. Available from www. ginaasthma.com.

23. Crapo RO, Casaburi R, Coates AL, et al: Guidelines for methacholine and exercise challenge testing-1999. This official statement of the American Thoracic Society was adopted by the ATS Board of Directors, July 1999. Am J Respir Crit Care Med 161: 309-329, 2000.

24. Standardization of Spirometry 1994 Update. American Thoracic Society. Am J Respir Crit Care Med 152: 1107-1136, 1995.

25. Morris JF: Spirometry in the evaluation of pulmonary function. West J Med 125: 110-118, 1976.

26. SensorMedics: Pulmonary Utilities Operator's Manual: Predicted Normal Equations. SensorMedics, Yorba Linda, 1988.

27. Cormican LJ, Farooque S, Altmann DR and Lee TH: Improvements in an oral aspirin challenge protocol for the diagnosis of aspirin hypersensitivity. Clin Exp Allergy 35: 717-722, 2005.

28. Kim TH, Chang HS, Park SM, et al: Association of angiotensin I-converting enzyme gene polymorphisms with aspirin intolerance in asthmatics. Clin Exp Allergy 38: 1727-1737, 2008.
29. Livak KJ: Allelic discrimination using fluorogenic probes and the 5' nuclease assay. Genet Anal 14: 143-149, 1999.

30. Stephens M, Smith NJ and Donnelly P: A new statistical method for haplotype reconstruction from population data. Am J Hum Genet 68: 978-989, 2001.

31. Barrett JC, Fry B, Maller J and Daly MJ: Haploview: analysis and visualization of LD and haplotype maps. Bioinformatics 21 263-265, 2005

32. Nyholt DR: A simple correction for multiple testing for singlenucleotide polymorphisms in linkage disequilibrium with each other. Am J Hum Genet 74: 765-769, 2004

33. Wakasugi $\mathrm{K}$ and Schimmel P: Two distinct cytokines released from a human aminoacyl-tRNA synthetase. Science 284: 147-151, 1999.

34. Hull J, Ackerman H, Isles K, et al: Unusual haplotypic structure of IL8, a susceptibility locus for a common respiratory virus. Am J Hum Genet 69: 413-419, 2001.

35. Hull J, Thomson A and Kwiatkowski D: Association of respiratory syncytial virus bronchiolitis with the interleukin 8 gene region in UK families. Thorax 55: 1023-1027, 2000

36. Kikuchi I, Kikuchi S, Kobayashi T, et al: Eosinophil transbasement membrane migration induced by interleukin- 8 and neutrophils. Am J Respir Cell Mol Biol 34: 760-765, 2006.

37. Gibson PG, Simpson JL and Saltos N: Heterogeneity of airway inflammation in persistent asthma: evidence of neutrophilic inflammation and increased sputum interleukin-8. Chest 119: 1329-1336, 2001.

38. Kleeman TA, Wei D, Simpson KL and First EA: Human tyrosyltRNA synthetase shares amino acid sequence homology with a putative cytokine. J Biol Chem 272: 14420-14425, 1997.

39. Kim SH, Ye YM, Lee SK, et al: Association of TNF-alpha genetic polymorphism with HLA DPB1*0301. Clin Exp Allergy 36: 1247-1253, 2006

40. Schroecksnadel K, Winkler C, Wirleitner B, Schennach H and Fuchs D: Aspirin down-regulates tryptophan degradation in stimulated human peripheral blood mononuclear cells in vitro. Clin Exp Immunol 140: 41-45, 2005. 